\title{
Circular RNA hsa_circ_0001380 in peripheral blood as a potential diagnostic biomarker for active pulmonary tuberculosis
}

\author{
HOU-LONG LUO ${ }^{1-3^{*}}$, YING PENG ${ }^{1,2^{*}}$, HONG LUO $^{1,2^{*}}$, JUN-AI ZHANG $^{1,2}$, GAN-BIN LIU ${ }^{4}$, HUAN XU $^{1,2}$, \\ GUI-XIAN HUANG ${ }^{2}$, YIN-FU SUN ${ }^{2}$, JI HUANG ${ }^{1,2}$, BI-YING ZHENG $^{1,2}$, JI-XIN ZHONG $^{5}$ and JUN-FA XU ${ }^{1,2}$ \\ ${ }^{1}$ Institute of Laboratory Medicine, Guangdong Medical University; ${ }^{2}$ Guangdong Provincial Key Laboratory of \\ Medical Molecular Diagnostics, Dongguan, Guangdong 523808; ${ }^{3}$ Department of Clinical Laboratory, \\ Peking University Shenzhen Hospital, Shenzhen, Guangdong 518036; ${ }^{4}$ Department of Respiration, \\ Dongguan Sixth Hospital, Dongguan, Guangdong 523008, P.R. China; ${ }^{5}$ Department of Medicine, \\ University of Maryland School of Medicine, Baltimore, MD 21201, USA
}

Received April 30, 2019; Accepted October 22, 2019

DOI: $10.3892 / \mathrm{mmr} .2020 .10992$

\begin{abstract}
Numerous studies have suggested that circular RNAs (circRNAs), a type of non-coding RNA lacking 5'-caps and 3'-poly(A) tails, are involved in the biological processes of various human diseases. However, little is known about their functions and diagnostic value in active pulmonary tuberculosis (APTB). The aim of the present study was to examine whether hsa_circ_0001380 is able to serve as a diagnostic biomarker for patients with APTB. The expression level of hsa_circ_0001380 was detected in the peripheral blood of 32 patients with APTB and 31 healthy volunteers by reverse transcription-quantitative PCR. The functional prediction of hsa_circ_0001380 was performed in silico. RNase R was used to detect the stability of hsa_circ_0001380. Finally, the diagnostic value of hsa_circ_0001380 was evaluated by receiver operating characteristic (ROC) curve analysis. The results showed that hsa_circ_0001380 was significantly downregulated in the peripheral blood of patients with APTB. In addition, hsa_circ_0001380 was found to be resistant to RNase R treatment. Moreover, four N6-adenosine methylation modification sites and two potential microRNA binding sites were predicted in silico. Importantly, the area under the ROC curve was 0.9502 , which suggested that hsa_circ_0001380 may act as a diagnostic biomarker for APTB. Taken together, the results indicated that circRNA hsa_circ_0001380 was downregulated in the peripheral blood of patients with APTB, and could serve as a diagnostic biomarker.
\end{abstract}

Correspondence to: $\mathrm{Dr} \mathrm{Jun}-\mathrm{Fa} \mathrm{Xu}$, Institute of Laboratory Medicine, Guangdong Medical University, 1 Xincheng Road, Dongguan, Guangdong 523808, P.R. China

E-mail: imxujunfa@163.com; xujunfa@gdmc.edu.cn

*Contributed equally

Key words: circular RNA, hsa_circ_0001380, tuberculosis, biomarker

\section{Introduction}

Tuberculosis (TB) remains a major global public health problem. According to the 2019 Global Tuberculosis Report, TB is one of the top 10 causes of death worldwide, followed by AIDS (1). In 2018, there were an estimated 1.2 million (range, 1.1-1.3 million) deaths from TB among HIV-negative people and an additional 251,000 (range, 223,000-281,000) deaths from TB among HIV-positive people. Additionally, an estimated 10.0 million (range, 9.0-11.1 million) people fell ill with TB, equivalent to 132 cases (range, 118-146) per 100,000 population (1). In order to improve TB management and reduce the transmission of Mycobacterium tuberculosis (Mtb), early diagnosis is essential in patients with active pulmonary TB (APTB) (2). However, traditional methods based on directly detecting $M t b$ do not meet clinical requirements due to their low sensitivity and specificity.

Circular RNAs (circRNAs), a novel type of non-coding RNA lacking 5' caps and 3'-poly(A) tails, have been recently reported to participate in the pathological processes of various human diseases (3-5). circRNAs can be divided into three categories according to their origin in genomic regions, namely exon circRNAs, intron circRNAs and exon-intron circRNAs (6-8). circRNAs are more stable than linear RNA and resist exonuclease RNase $\mathrm{R}$ digestion due to their closed loop structure (9). Their expression is often tissue-specific and developmental-stage specific $(7,10)$. These properties suggest that circRNAs are an ideal biomarker for disease diagnosis.

Recent studies have reported that circRNAs may serve as potential biomarkers for a number of human diseases. For example, Bao et al (11) indicated that hsa_circ_0037911 in whole blood samples could be a stable biomarker for early diagnosis of essential hypertension. Hang et al (12) suggested that a novel plasma circRNA, circFARSA, may be a potential biomarker for non-small cell lung cancer. Zhao et al (13) found that hsa_circ_0001275 in peripheral blood mononuclear cells (PBMCs) could serve as a potential novel diagnostic biomarker for postmenopausal osteoporosis. Nevertheless, whether circRNAs could act as novel biomarkers for APTB diagnosis and therapeutic prediction remains unknown. 
Table I. Primer sequences for reverse transcription-quantitative PCR analysis.

\begin{tabular}{llc}
\hline Name & \multicolumn{1}{c}{ Primer sequence $\left(5^{\prime} \rightarrow 3^{\prime}\right)$} & Product length $(\mathrm{bp})$ \\
\hline hsa_circ_0001380 & F: TTCCGGCCACCCATTGATTT & 132 \\
& R: GGCCGTCGTCTTTTAGGAGC & 224 \\
GAPDH & F: GAAGGTCGGAGTCAACGGATT & \\
& R: CCTGGAAGATGGTGATGGGATT & \\
\hline
\end{tabular}

F, forward; R, reverse.

In our previous study, circRNA-sequencing (seq) in PBMCs revealed differential expression of circRNAs in patients with APTB (14). In the present study, only hsa_circ_0001380 was evaluated. The results revealed that hsa_circ_0001380 was significantly downregulated in the PBMCs of patients with APTB compared with healthy volunteers (HVs). hsa circ_0001380 is located at chr3 196,118,683-196,129,890 with a spliced length of $247 \mathrm{nt}$, and is derived from exons 3-5 of the UBXN7 gene. In addition, the potential functions of hsa circ_0001380 were explored in silico. A receiver operating characteristic (ROC) curve was also constructed to evaluate the diagnostic value of hsa_circ_0001380 in APTB. These results indicated that hsa_circ_0001380 may be a potential diagnostic biomarker for APTB.

\section{Materials and methods}

Patients and clinical samples. In the present study, a total of 32 patients with APTB (22 male, 10 female), aged 17-60 years, were recruited from Guangdong Medical University and Dongguan Sixth Hospital from February 2018 to August 2018, as previously described (14). APTB was detected in all subjects by Ziehl-Neelsen acid fast staining of the sputum smears and Lowenstein-Jensen slant culture according to the 'Health Standards for the People's Republic of China-Diagnosis of Tuberculosis' (WS 288-2017) (15). The diagnosis was based on sputum smear examination and clinical symptoms. A total of $31 \mathrm{HVs}$ (20 male, 11 female) aged 20-65 years, with no bacteriological or clinical evidence of APTB, were recruited as controls from February 2018 to August 2018. A volume of $\sim 5 \mathrm{ml}$ peripheral blood was collected at the different time points (patients with TB within $24 \mathrm{~h}$ of admission to the hospital and $\mathrm{HV}$ at 6:00-8:00 am.) from each subject. Ethical approval was obtained from the Ethical Committee of Dongguan Sixth Hospital, Guangdong Medical University. All subjects provided written informed consent prior to the study.

Isolation of PBMCs. PBMCs were isolated as previously reported (16). Briefly, PBMCs were freshly isolated from peripheral blood by density gradient centrifugation on Ficoll-Paque (TBD Science) at $450 \mathrm{x}$ g for $20 \mathrm{~min}$ at $20^{\circ} \mathrm{C}$, according to the protocol of the manufacturer. Cell viability was tested using the Trypan blue dye exclusion method ( $>95 \%$ in all experiments). Next, the PBMCs were lysed with TRIzol ${ }^{\circledR}$ reagent (Invitrogen; Thermo Fisher Scientific, Inc.) and stored at $-80^{\circ} \mathrm{C}$ for further experiments.
Table II. Clinical data for all study subjects.

\begin{tabular}{lcc}
\hline Characteristic & $\begin{array}{c}\text { APTB group } \\
(\mathrm{n}=32)\end{array}$ & $\begin{array}{c}\text { HV group } \\
(\mathrm{n}=31)\end{array}$ \\
\hline Age, years; mean \pm SD (range) & $\begin{array}{c}36.03 \pm 13.96 \\
(17-60)\end{array}$ & $\begin{array}{c}33.26 \pm 14.56 \\
(20-65)\end{array}$ \\
Sex, $\mathrm{n}$ & & \\
Male & 22 & 20 \\
Female & 10 & 11 \\
Sputum smear, $\mathrm{n}$ & & \\
Positive & 25 & 0 \\
Negative & 7 & 31 \\
\hline
\end{tabular}

APTB, active pulmonary tuberculosis patients; HV, healthy volunteers.

RNA sample preparation and quality control. Total RNA was extracted from the PBMCs using TRIzol ${ }^{\circledR}$ reagent (Invitrogen; Thermo Fisher Scientific, Inc.) according to the instructions of the manufacturer. The quantity of total RNA was determined at 260/280 nm using NanoDrop ND-2000 spectrophotometer (Thermo Fisher Scientific,Inc.). The RNA integrity was evaluated by $2 \%$ agarose gel electrophoresis, and the bands were visualized using nucleic acid dye ethidium bromide (data not shown).

RNase $R$ digestion. To assess the stability of circRNAs, exonuclease RNase R (Epicentre; Illumina, Inc.) was used to treat the total RNA, according to the protocol of the manufacturer. Briefly, $2 \mu \mathrm{g}$ total RNA was digested with or without $2 \mathrm{U}$ RNase $\mathrm{R}$ for $10 \mathrm{~min}$ at $37^{\circ} \mathrm{C}$ in $1 \mathrm{X}$ RNase $\mathrm{R}$ reaction buffer (Epicentre; Illumina, Inc.), and reverse transcription-quantitative (RT-q)PCR was subsequently performed.

$R T$ - $q P C R$. A total of $500 \mathrm{ng}$ purified total RNA was used for cDNA synthesis. Total RNA was reverse transcribed into cDNA at $37^{\circ} \mathrm{C}$ for $15 \mathrm{~min}$ and $85^{\circ} \mathrm{C}$ for $5 \mathrm{sec}$ using a Primescript RT reagent kit with random primers according to the protocol of the manufacturer (Takara Bio, Inc.). Next, qPCR was performed using the Applied Biosystems QuantStudio 6 Flex System (Thermo Fisher Scientific, Inc.) with TransStart Tip Green qPCR SuperMix (Beijing Transgen Biotech Co., Ltd.) at $95^{\circ} \mathrm{C}$ for $2 \mathrm{~min}$, followed by 40 cycles of $95^{\circ} \mathrm{C}$ for $5 \mathrm{sec}, 60^{\circ} \mathrm{C}$ for $30 \mathrm{sec}, 72^{\circ} \mathrm{C}$ for $30 \mathrm{sec}$. Also, the melting curve of RT-qPCR was used to evaluate the specificity of the amplification 

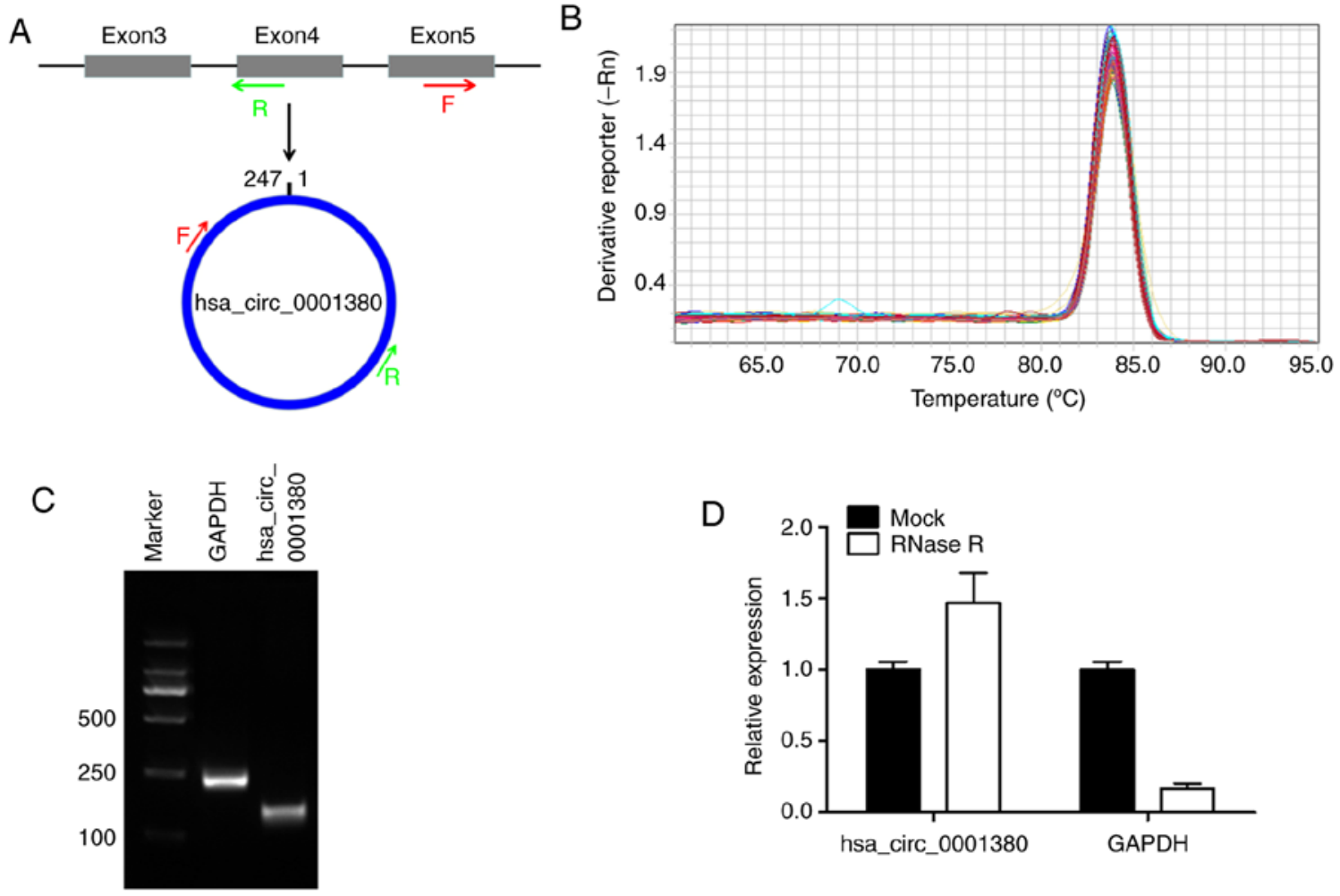

Figure 1. Validation and characteristics of hsa_circ_0001380 in human peripheral blood mononuclear cells. (A) Pattern of hsa_circ_0001380 formation and design of divergent primers. Red arrows indicate the forward primer and green arrows indicate the reverse primer. (B) Melting curve analysis of hsa circ_0001380, constructed via RT-qPCR. (C) Agarose gel electropherogram of the RT-qPCR product. Lanes 1, 2 and 3 correspond to the DNA marker, GAPDH and hsa_circ_0001380, respectively. (D) RT-qPCR analysis of hsa_circ_0001380 and GAPDH after treatment with (RNase R) or without RNase R (Mock). RT-qPCR, reverse transcription-quantitative polymerase chain reaction; F, forward; R, reverse.

product using the software of QuantStudio 6 Flex Real-time PCR System. In order to detect its specific expression, divergent primers crossing the back-splice junction were designed. The primer sequences used in the present study are listed in Table I. The primers were synthesized by Sangon Biotech Co., Ltd. GAPDH was used as an internal control. All RT-qPCR reactions were performed in triplicate. The data were analyzed using the $2^{-\Delta \Delta C q}$ method (17) to calculate the relative expression. The PCR product was visualized by used $2 \%$ agarose gel with ethidium bromide nucleic acid dye added $\beta$.

Function prediction of hsa_circ_0001380. The CircBank database (http://www.circbank.cn/) was used to search circRNA basic information, retrieve circRNA sequence, and predict circRNA protein coding potential, circRNA conservation and circRNA modification. The interactions between circRNA and miRNAs were predicted using the CircBank and Circinteractome databases (https://circinteractome.nia.nih.gov/).

Statistical analysis. Receiver operating characteristic (ROC) curve analysis was conducted to assess the clinical diagnostic ability of hsa_circ_0001380. Each value is represented as the mean \pm SEM. Data analysis was performed using the Student's t-test or one-way ANOVA with Tukey's test for continuous variables and $\chi^{2}$ test for discontinuous variables, and $\mathrm{P}<0.05$ was considered to indicate a statistically significant difference. The statistical analyses were performed using GraphPad Prism (version 5.0; GraphPad Software, Inc.) and SPSS software (version 17.0; SPSS, Inc.).

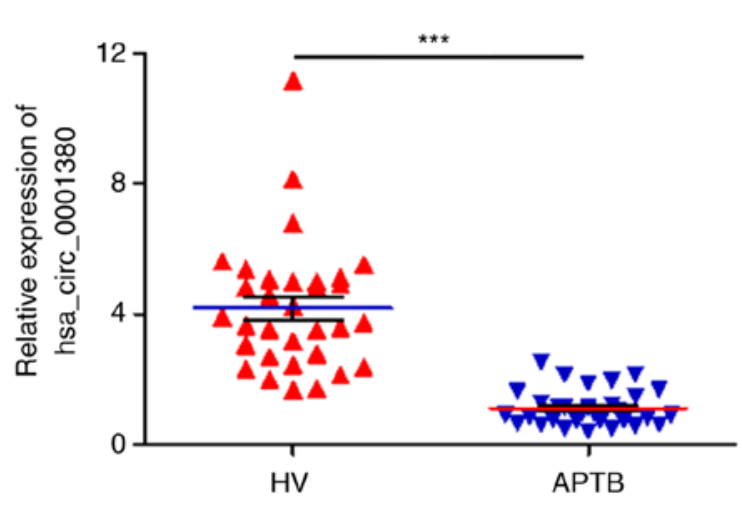

Figure 2. Relative expression of hsa_circ_0001380 in the peripheral blood mononuclear cells of patients with APTB and HVs assessed by reverse transcription-quantitative polymerase chain reaction analysis. ${ }^{* * *} \mathrm{P}<0.001$ APTB, active pulmonary tuberculosis; $\mathrm{HV}$, healthy volunteer.

\section{Results}

Clinical characteristics of all study subjects. In the present study, prospective clinical data were collected from 32 patients with APTB and $31 \mathrm{HV}$. The demographic and clinical characteristics of all study subjects are displayed in Table II. No statistically significant age- and sex-related differences were observed between patients with APTB and HV $(\mathrm{P}>0.05)$. Among patients with APTB, Ziehl-Neelsen acid fast staining analysis of the sputum smears revealed that $78.12 \%$ of samples were $M t b$ positive. 
A

Basic information

circBank ID: hsa_circUBXN7_006

circBase ID: hsa_circ_0001380

Position: chr3: 196118683-196129890 strand: -

Length: 247
Host gene symbol: UBXN7

bestTranscript: NM_015562

Annotation: ANNOTATED, CDS, coding, INTERNAL, OVCODE,

OVEXON

coding_potential_assessment

circBase ID: hsa_circ_0001380

circRNA_size: 247

ORF_size: 51
Fickett_score: 0.9308

Hexamer score: -0.1876

coding_prob: 0.0054

\section{RNA modification}

\begin{tabular}{|l|l|l|l|l|l|}
\hline chr & Start & End & Name & Strand & m6A levels \\
\hline chr3 & $1961186 \ldots$ & $196120 \ldots$ & chr3junc10_R2_A & - & 0.1763 \\
\hline chr3 & $1961186 \ldots$ & $196129 \ldots$ & chr3junc58_R2_A_chr3junc94_R2 & - & 0.0996 \\
\hline chr3 & $1961186 \ldots$ & $196120 \ldots$ & chr3junc33_R1_B & - & 0.0961 \\
\hline chr3 & $1961186 \ldots$ & $196129 \ldots$ & chr3junc77_R1_B & - & 0.0961 \\
\hline
\end{tabular}

Showing 1 to 4 of 4 rows

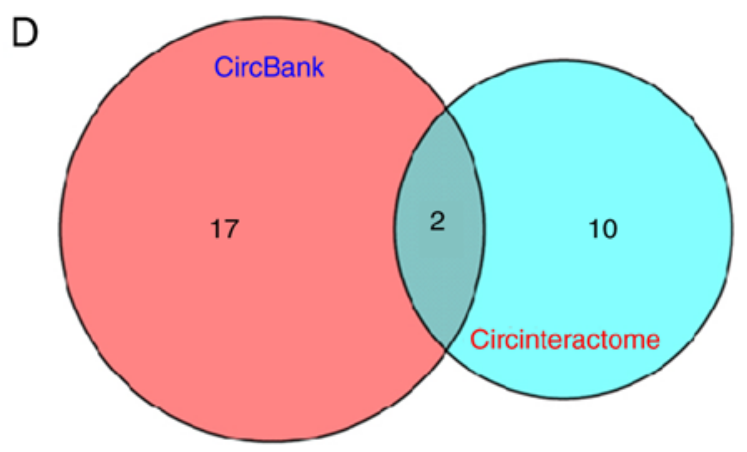

E

\begin{tabular}{|c|c|c|c|c|c|c|c|c|c|c|c|}
\hline $\begin{array}{l}\text { CircRNA } \\
\text { Mirbasa ID }\end{array}$ & $\begin{array}{l}\text { CircRNA (Top)-miRNA } \\
\text { (Bottom) pairing }\end{array}$ & $\begin{array}{l}\text { Site } \\
\text { type }\end{array}$ & $\begin{array}{c}\text { CircRNA } \\
\text { start }\end{array}$ & $\begin{array}{c}\text { CircRNA } \\
\text { End }\end{array}$ & $\begin{array}{c}3^{\prime} \\
\text { pairing }\end{array}$ & $\begin{array}{l}\text { Local } \\
\mathrm{AU}\end{array}$ & Position & TA & SPS & $\begin{array}{c}\text { Context+ } \\
\text { score }\end{array}$ & $\begin{array}{l}\text { Context+ } \\
\text { score } \\
\text { percentile }\end{array}$ \\
\hline $\begin{array}{l}\text { hsa_dirc_0001380 }\left(5^{\prime} \ldots 3^{\prime}\right) \\
\text { hsa-miR-136 (3'..5) }\end{array}$ & $\begin{array}{r}\text { GAACAAGAAUUAAGAAAUGGAGG } \\
11111111 \\
\text { AGGUAGUAGUUUUGUUUACCUCA }\end{array}$ & 7mer-m8 & 158 & 164 & 0.003 & -0.041 & -0.050 & 0.008 & 0.017 & -0.183 & 93 \\
\hline $\begin{array}{l}\text { hsa_circ_0001380 }\left(5^{\prime} \ldots 3^{3}\right) \\
\text { hsa -miR-622 }\left(3^{\prime} \ldots 5^{\prime}\right)\end{array}$ & $\begin{array}{r}\text { GGUUUCCGGGAUUUUCAGACUGA } \\
11111111 \\
\text { CGAGGUUGGAGUCGUCUGACA }\end{array}$ & 8mer-1a & 122 & 129 & 0.013 & -0.062 & -0.084 & 0.014 & -0.036 & -0.402 & 99 \\
\hline
\end{tabular}

Figure 3. Functions of hsa_circ_0001380 predicted in silico. hsa_circ_0001380 information as predicted in the CircBank database. (A) Basic information (B) Coding potential assessment. (C) m6A modification sites. (D) Venn diagram displaying the potential miRNAs associated with hsa_circ_0001380 predicted by the CircBank and Circinteractome databases. (E) Interaction between hsa_circ_0001380 and miR-622/miR136 was predicted by Circinteractome, including the circRNA-miRNA pairing site, site type and context+score percentile. m6A, N6-adenosine methylation.

Validation of hsa_circ_0001380. hsa_circ_0001380 was derived from exon $3-5$ of the UBXN7 gene locus by back-splicing. In order to detect its specific expression, divergent primers crossing the back-splice junction were designed (Fig. 1A). The melting curve analysis of RT-qPCR revealed a single peak, demonstrating the specificity of the 
Table III. Relevant indices of diagnostic experiment.

\begin{tabular}{lccccc}
\hline circRNA & AUC & Sensitivity & Specificity & Total consistent rate & Youden index \\
\hline Hsa_circ_0001380 & 0.95 & $93.75 \%$ & $87.50 \%$ & $90.62 \%$ & 0.81
\end{tabular}

AUC, area under the receiver operating characteristic curve; circRNA, circular RNA.

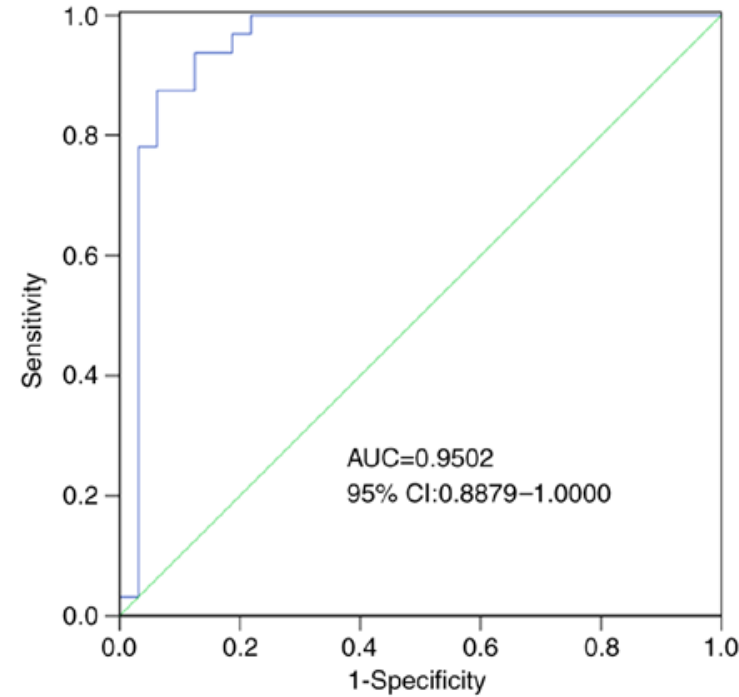

Figure 4. A ROC curve was constructed to assess the potential diagnostic value of hsa_circ_0001380. The AUC was 0.9502 (95\% CI: 0.8879-1.0000) $\mathrm{CI}$, confidence interval; ROC, receiver operating characteristic; AUC, area under the curve.

amplification product (Fig. 1B). The size of the amplification product was evaluated by agarose gel electrophoresis and was consistent with its full size (Fig. 1C). Subsequently, RNase $\mathrm{R}$ was used to detect the stability of hsa_circ_0001380, and the results indicated that hsa_circ_0001380 was stable in tolerance to RNase enzyme treatment. However, the control linear mRNA GAPDH could not tolerate RNAase treatment (Fig. 1D). These results demonstrated that hsa_circ_0001380 in human PBMCs could be specifically amplified by RT-qPCR. Additionally, before RT-qPCR analysis, the RNA integrity was evaluated and visualized using $2 \%$ agarose gel added ethidium bromide dye (data not shown).

hsa_circ_0001380was downregulated in APTB. In our previous circRNA sequencing study, a large number of differential circRNAs were identified in the PBMCs of patients with APTB (14). Of note, hsa_circ_0001380 was revealed as one of these differential circRNAs, and the fold-change between healthy participants and APTB patients was found to be $\sim 1.6$. In order to validate the expression level of hsa circ_0001380 in APTB, PBMCs from 32 patients with APTB and $31 \mathrm{HV}$ were collected and analyzed by RT-qPCR, using GAPDH as an internal control. The results demonstrated that hsa_circ_0001380 expression was significantly downregulated in the PBMCs of patients with APTB as compared with the HV group $(\mathrm{P}<0.001$; Fig. 2$)$. This finding suggested that hsa_circ_0001380 may be related to tuberculosis infection.
Functions of hsa_circ_0001380 predicted in silico. CircBank database analysis indicated that hsa_circ_0001380 is located in chr3 $(196,118,683-196,129,890)$, has a length of $247 \mathrm{nt}$, consists of three exons (exons 3-5) from the UBXN7 gene locus and its CircBank ID is hsa_circUBXN7_006 (Fig. 3A). Since circRNAs are able to exert their biological functions through multiple pathways, the functions of hsa_circ_0001380 were predicted in silico. Although certain circRNAs are capable of translating proteins or polypeptides, hsa_circ_0001380 has little translation potential (Fig. 3B) according to the prediction of coding potential assessment based on the CircBank database. The N6-adenosine methylation (m6A) modification serves an important role in RNA modification (18), thus m6A expression in hsa_circ_0001380 was also predicted. The results demonstrated that hsa_circ_0001380 has four m6A modification sites (Fig. 3C). To investigate the potential microRNAs (miRNAs/miRs) associated with hsa_circ_0001380, the CircBank and Circinteractome databases were used based on the TargetScan and miRanda algorithms (Fig. 3D). The two most potentially complementary binding miRNAs, miR-136 and miR-622, are presented in Fig. 3E.

Diagnostic potential of hsa_circ_0001380 in APTB. Numerous previous studies have reported that circRNAs may be effective biomarkers for disease diagnosis (11-13). The results indicated that hsa_circ_0001380 was downregulated in APTB and was more stable than linear RNA. Therefore, hsa_circ_0001380 may act as a promising biomarker for APTB. To assess the diagnostic value of hsa_circ_0001380, ROC analysis was conducted. The results revealed that the area under the ROC curve (AUC) was 0.9502 (95\% confidence interval, 0.8879-1.0000), which indicated the high diagnostic value of hsa_circ_0001380 in APTB. The sensitivity, specificity, total consistent rate, and Youden's index were 93.75, $87.50,90.62 \%$ and 0.81 , respectively (Fig. 4 and Table III).

\section{Discussion}

Tuberculosis, which is caused by $M t b$, remains a major public health problem worldwide. The emergence of drug-resistant tuberculosis, tuberculosis concomitant with HIV infection, and the current lack of techniques for its early diagnosis have led to the global spread of tuberculosis (1). Accurate and early diagnosis and detection of drug-sensitive and drug-resistant tuberculosis is essential for achieving tuberculosis control worldwide (19). In addition to traditional methods based on $M t b$ detection, molecular biological techniques based on $M t b$-specific genomic DNA and immunological methods, a number of novel techniques have also been utilized to detect Mtb (20). For example, electrochemical (21), optical (22) and 
magnetic (23) detection techniques based on biosensing technology have been used to detect $M t b$. However, these methods fail to meet clinical needs due to their low sensitivity and specificity.

Previous studies have indicated that circRNAs are abundant and stable non-coding RNAs lacking $5^{\prime}$ and $3^{\prime}$ termini that are widely present in diverse bodily fluids, including plasma/serum (24), blood (25) and saliva (26). Therefore, circRNAs may represent a potential diagnostic biomarker for human diseases. Numerous studies have indicated that circRNAs are efficient biomarkers for human diseases, including bladder cancer (27). hepatocellular carcinoma (28). glioma (29) and systemic lupus erythematosus (30).

In the present study, the potential diagnostic value of hsa_circ_0001380 was explored in the PBMCs of patients with APTB. The expression of hsa_circ_0001380 was significantly decreased in APTB. A ROC curve was constructed to assess the diagnostic value of hsa_circ_0001380, and the results showed that the AUC was 0.9502 and sensitivity and specificity were 93.75 and $87.50 \%$, respectively. These results suggested that hsa_circ_0001380 may serve as an efficient biomarker for APTB. Future studies with larger clinical sample sizes and additional disease control groups are required to further understand the role of hsa_circ_0001380.

circRNAs may exert their biological functions in various ways, affecting the occurrence and development of human diseases. Numerous studies have proposed that circRNAs act as competing endogenous RNA, which compete with miRNA binding to mRNAs. For example, Cheng et al (4) reported that circRNA VMA21 prevented intervertebral disc degeneration by targeting miR-200c and the X-linked inhibitor of apoptosis protein. In the present study, hsa_circ_0001380 was matched with hsa-miR-622 and hsa-miR-136-5p based on TargetScan and miRanda algorithms. In addition, Zhou et al (18) demonstrated that the m6A modification is widespread in circRNAs and m6A circRNAs are expressed in cell-type-specific patterns. The bioinformatics results showed that hsa_circ_0001380 has four m6A modification sites. Hence, hsa_circ_0001380 may exert its biological function via m6A modification. Moreover, certain circRNAs have short open reading frames and/or internal ribosome entry sites, and are able to translate proteins or polypeptides (31-33). However, hsa_circ_0001380 was found to have little translation potential in silico.

The present study has several limitations. First, the sample size was limited. Nevertheless, since the statistical analysis results were considered statistically significant, the sample size was not expanded. Secondly, the present study was only a preliminary exploration of hsa_circ_0001380 as a diagnostic marker for APTB. At present, to the best of the authors' knowledge, there is no similar study on hsa_circ_0001380 in TB. In the future, its specific molecular function should be further explored by loss-of-function and gain-of-function analyses in TB.

In conclusion, the present study suggested that the expression of hsa_circ_0001380 was significantly downregulated in the PBMCs of patients with APTB as compared with HV. The dysregulation of hsa_circ_0001380 expression indicated that it may be involved in the occurrence and development of APTB. More importantly, hsa_circ_0001380 may serve as a novel potential diagnostic biomarker for APTB.

\section{Acknowledgements}

Not applicable.

\section{Funding}

The present study was supported by The National Natural Science Foundation of China (grant nos. 81870016, 81570009 and 81273237), The Natural Science Foundation of Guangdong Province (grant no. 2015A030313513) and The Science and Technology Innovation Fund of Guangdong Medical University (grant nos. STIF201110 and B2012078).

\section{Availability of data and materials}

The datasets used and/or analyzed in the present study are available from the corresponding author on reasonable request.

\section{Authors' contributions}

JX, HLL, YP, JAZ, BZ and JH conceived and designed the study. HLL, YP and HL performed the experiments. HLL, YP, GL, HX, GH, YS, JXZ and JX performed data collection and interpretation. HLL, YP, JXZ and BZ prepared the figures and drafted the manuscript. JX, JXZ and BZ revised the manuscript. HLL, JX and JH reviewed and edited the manuscript. All authors read and approved the final manuscript.

\section{Ethics approval and consent to participate}

The present study was approved by the Ethical Committee of Dongguan Sixth Hospital, Guangdong Medical University. Experimental procedures were implemented in accordance with the guidelines and regulations of Guangdong Medical University and written informed consent was obtained from all patients and healthy blood donors.

\section{Patient consent for publication}

Not applicable.

\section{Competing interests}

The authors declare that they have no competing interests.

\section{References}

1. WHO: Global tuberculosis report 2019. Geneva: World Health Organization, 2017.

2. Floyd K, Glaziou P, Zumla A and Raviglione M: The global tuberculosis epidemic and progress in care, prevention, and research: An overview in year 3 of the End TB era. Lancet Respir Med 6: 299-314, 2018

3. Yang L, Han B, Zhang Y, Bai Y, Chao J, Hu G and Yao H: Engagement of circular RNA HECW2 in the nonautophagic role of ATG5 implicated in the endothelial-mesenchymal transition. Autophagy 14: 404-418, 2018.

4. Cheng X, Zhang L, Zhang K, Zhang G, Hu Y, Sun X, Zhao C, Li H, $\mathrm{Li}$ YM and Zhao J: Circular RNA VMA21 protects against intervertebral disc degeneration through targeting miR-200c and X linked inhibitor-of-apoptosis protein. Ann Rheum Dis 77: 770-779, 2018.

5. Qiu M, Xia W, Chen R, Wang S, Xu Y, Ma Z, Xu W, Zhang E, Wang J, Fang T, et al: The circular RNA circPRKCI promotes tumor growth in lung adenocarcinoma. Cancer Res 78: 2839-2851, 2018. 
6. Zhang Y, Zhang XO, Chen T, Xiang JF, Yin QF, Xing YH, Zhu S, Yang $L$ and Chen LL: Circular intronic long noncoding RNAs. Mol Cell 51: 792-806, 2013.

7. Memczak S, Jens M, Elefsinioti A, Torti F, Krueger J, Rybak A, Maier L, Mackowiak SD, Gregersen LH, Munschauer M, et al Circular RNAs are a large class of animal RNAs with regulatory potency. Nature 495: 333-338, 2013.

8. Chen L, Huang C, Wang X and Shan G: Circular RNAs in eukaryotic cells. Curr Genomics 16: 312-318, 2015.

9. Suzuki $H$ and Tsukahara T: A view of pre-mRNA splicing from RNase R resistant RNAs. Int J Mol Sci 15: 9331-9342, 2014.

10. Salzman J, Chen RE, Olsen MN, Wang PL and Brown PO Cell-type specific features of circular RNA expression. PLoS Genet 9: e1003777, 2013

11. Bao X, Zheng S, Mao S, Gu T, Liu S, Sun J and Zhang L: A potential risk factor of essential hypertension in case-control study: Circular RNA hsa_circ_0037911. Biochem Biophys Res Commun 498: 789-794, 2018

12. Hang D, Zhou J, Qin N, Zhou W, Ma H, Jin G, Hu Z, Dai J and Shen H: A novel plasma circular RNA circFARSA is a potential biomarker for non-small cell lung cancer. Cancer Med 7: 2783-2791, 2018

13. Zhao K, Zhao Q, Guo Z, Chen Z, Hu Y, Su J, Chen L, He Z, Cai X, Chen M, et al: Hsa_Circ_0001275: A potential novel diagnostic biomarker for postmenopausal osteoporosis. Cell Physio Biochem 46: 2508-2516, 2018.

14. Zhuang ZG, Zhang JA, Luo HL, Liu GB, Lu YB, Ge NH, Zheng BY, Li RX, Chen C, Wang X, et al: The circular RNA of peripheral blood mononuclear cells: Hsa_circ_0005836 as a new diagnostic biomarker and therapeutic target of active pulmonary tuberculosis. Mol Immunol 90: 264-272, 2017.

15. National Health Commission of the People's Republic of China WS 288-2017 diagnosis for pulmonary tuberculosis[S]. Beijing: China Standards Press, 2017.

16. Zeng JC, Lin DZ, Yi LL, Liu GB, Zhang H, Wang WD, Zhang JA, Wu XJ, Xiang WY, Kong B, et al: BTLA exhibits immune memory for alphabeta $\mathrm{T}$ cells in patients with active pulmonary tuberculosis. Am J Transl Res 6: 494-506, 2014.

17. Livak KJ and Schmittgen TD: Analysis of relative gene expression data using real-time quantitative PCR and the 2(-Delta Delta C(T)) methods. Methods 25: 402-408, 2001

18. Zhou C, Molinie B, Daneshvar K, Pondick JV, Wang J, Van Wittenberghe N, Xing Y, Giallourakis CC and Mullen AC: Genome-Wide maps of m6A circRNAs identify widespread and cell-type-specific methylation patterns that are distinct from mRNAs. Cell Rep 20: 2262-2276, 2017.

19. Walzl G, Mcnerney R, du Plessis N, Bates M, McHugh TD, Chegou NN and Zumla A: Tuberculosis: Advances and challenges in development of new diagnostics and biomarkers. Lancet Infect Dis 18: e199-e210, 2018

20. Gupta S and Kakkar V: Recent technological advancements in tuberculosis diagnostics-A review. Biosens Bioelectron 115: 14-29, 2018.

21. Pavlou AK, Magan N, Jones JM, Brown J, Klatser P and Turner AP: Detection of Mycobacterium tuberculosis (TB) in vitro and in situ using an electronic nose in combination with a neural network system. Biosens Bioelectron 20: 538-544, 2004.
22. Duman M and Piskin E: Detection of Mycobacterium tuberculosis complex and Mycobacterium gordonae on the same portable surface plasmon resonance sensor. Biosens Bioelectron 26: 908-912, 2010

23. Lee H, Yoon TJ and Weissleder R: Ultrasensitive detection of bacteria using core-shell nanoparticles and an NMR-filter system. Angew Chem Int Ed Engl 48: 5657-5660, 2009.

24. Koh W, Pan W, Gawad C, Fan HC, Kerchner GA, Wyss-Coray T, Blumenfeld YJ, El-Sayed YY and Quake SR: Noninvasive in vivo monitoring of tissue-specific global gene expression in humans. Proc Natl Acad Sci USA 111: 7361-7366, 2014.

25. Memczak S, Papavasileiou P, Peters O and Rajewsky N: Identification and characterization of circular RNAs as a new class of putative biomarkers in human blood. PLoS One 10: e141214, 2015.

26. Bahn JH, Zhang Q, Li F, Chan TM, Lin X, Kim Y, Wong DT and Xiao X: The landscape of microRNA, Piwi-interacting RNA, and circular RNA in human saliva. Clin Chem 61: 221-230, 2015

27. Yang C, Yuan W, Yang X, Li P, Wang J, Han J, Tao J, Li P, Yang H, Lv Q and Zhang W: Circular RNA circ-ITCH inhibits bladder cancer progression by sponging miR-17/miR-224 and regulating p21, PTEN expression. Mol Cancer 17: 19, 2018.

28. Chen G, Shi Y, Liu M and Sun J: circHIPK3 regulates cell proliferation and migration by sponging miR-124 and regulating AQP3 expression in hepatocellular carcinoma. Cell Death Dis 9: $175,2018$.

29. Li G, Yang H, Han K, Zhu D, Lun P and Zhao Y: A novel circular RNA, hsa_circ_0046701, promotes carcinogenesis by increasing the expression of miR-142-3p target ITGB8 in glioma. Biochem Biophys Res Commun 498: 254-261, 2018.

30. Wang X, Zhang C, Wu Z, Chen Y and Shi W: CircIBTK inhibits DNA demethylation and activation of AKT signaling pathway via miR-29b in peripheral blood mononuclear cells in systemic lupus erythematosus. Arthritis Res Ther 20: 118, 2018

31. Zhang M, Huang N, Yang X, Luo J, Yan S, Xiao F, Chen W, Gao X, Zhao K, Zhou H, et al: A novel protein encoded by the circular form of the SHPRH gene suppresses glioma tumorigenesis. Oncogene 37: 1805-1814, 2018.

32. Pamudurti NR, Bartok O, Jens M, Ashwal-Fluss R, Stottmeister C, Ruhe L, Hanan M, Wyler E, Perez-Hernandez D, Ramberger E, et al: Translation of circRNAs. Mol Cell 66: 9-21 e7, 2017.

33. Legnini I, Di Timoteo G, Rossi F, Morlando M, Briganti F, Sthandier O, Fatica A, Santini T, Andronache A, Wade M, et al: Circ-ZNF609 is a circular RNA that can be translated and functions in myogenesis. Mol Cell 66: 22-37, 2017.

This work is licensed under a Creative Commons Attribution-NonCommercial-NoDerivatives 4.0 International (CC BY-NC-ND 4.0) License. 